\title{
IL-6, Antioxidant Capacity and Muscle Damage Markers Following High-Intensity Interval Training Protocols
}

\author{
by \\ Lukas Cipryan ${ }^{1}$
}

The aim of this study was to investigate changes of interleukin-6 (IL-6), total antioxidant capacity (TAC) and muscle damage markers (creatine kinase (CK), myoglobin and lactate dehydrogenase (LDH)) in response to three different high-intensity interval training (HIIT) protocols of identical external work. Twelve moderately-trained males participated in the three HIIT trials which consisted of a warm-up, followed by $12 \mathrm{~min}$ of $15 \mathrm{~s}, 30 \mathrm{~s}$ or $60 \mathrm{~s}$ HIIT sequences with the work/rest ratio 1 . The biochemical markers of inflammation, oxidative stress and muscle damage were analysed POST, $3 \mathrm{~h}$ and $24 \mathrm{~h}$ after the exercise. All HIIT protocols caused an immediate increase in IL-6, TAC, $C K$, myoglobin and LDH. The most pronounced between-trials differences were found for the POST-exercise changes in IL-6 (Effect size $\pm 90 \%$ confidence interval: $1.51 \pm 0.63,0.84 \pm 0.34$ and $1.80 \pm 0.60$ for the $15 \mathrm{~s} / 15 \mathrm{~s}$, 30s/30s and $60 \mathrm{~s} / 60 \mathrm{~s}$ protocol, respectively) and myoglobin $(1.11 \pm 0.29,0.45 \pm 0.48$ and $1.09 \pm 0.22$ for the $15 \mathrm{~s} / 15 \mathrm{~s}, 30 \mathrm{~s} / 30 \mathrm{~s}$ and $60 \mathrm{~s} / 60 \mathrm{~s}$ protocol, respectively). There were no substantial between-trial differences in other biochemical variables. In conclusion, the $15 \mathrm{~s} / 15 \mathrm{~s}$ and $60 \mathrm{~s} / 60 \mathrm{~s}$ protocols might be preferred to the 30s/30s protocols in order to maximize the training stimulus.

Key words: inflammation, oxidative stress, creatine kinase, myoglobin, lactate dehydrogenase.

\section{Introduction}

High-intensity interval training (HIIT) ranks among the most popular training methods, mainly due to its time-efficiency and superior performance improvements when compared to traditional moderate-intensity continuous training (Milanović et al., 2015). Repeating the highintensity bouts of exercise enables the accumulation of extensive time spent at or close to $\dot{V} \mathrm{O}_{2 \max }$ intensity which is the necessary prerequisite for cardiorespiratory and metabolic adaptation to exercise training (Heydari et al., 2013). Achievement of the sufficient intensity can be carried out in numerous ways and is based on the manipulation of up to nine variables, including work and rest interval intensity and duration, exercise modality, the number of repetitions and sets, as well as between-sets recovery duration and intensity. The physiological response and adaptation to HIIT may therefore substantially differ and is still a matter of debate (Buchheit and Laursen, 2013; Tschakert et al., 2015).

Exercise-induced inflammation may play an important role in metabolic and musculoskeletal adaptation to exercise. It has been shown that skeletal muscle produces so-called myokines that might be responsible for the beneficial effect of exercise on muscle hypertrophy and myogenesis; moreover, it could also have a systemic effect on the liver, adipose tissue, immune and vascular system (Pedersen and Febbraio, 2012).

Interleukin-6 (IL-6) might be quoted as an example of one of these myokines. IL-6 is

1 - Human Motion Diagnostic Centre, Ostrava University, Czech Republic. 
predominantly considered a pro-inflammatory cytokine involved in many pathophysiological conditions (Pal et al., 2014; Yudkin, 2007). However, the release of IL-6 by contracting skeletal muscle seems to have a beneficial effect on insulin-stimulated glucose disposal and fatty acid oxidation. IL-6 might be, therefore, important for the muscle metabolism during contraction (Raschke and Eckel, 2013). The post-exercise changes in circulating IL-6 mainly correspond to the increase in net IL-6 release from muscle (Bernecker et al., 2013) and depend on exercise duration, intensity and mode (Febbraio and Pedersen, 2002). While there is some evidence that HIIT significantly influences the post-exercise IL-6 response (Wadley et al., 2016; Zweetsloot et al., 2014), the effects of various forms of HIIT have not been completely established as yet.

Cytokine production has also been associated with oxidative stress (Wadley et al., 2013). Oxidative stress is defined as an excessive production of reactive oxygen and nitrogen species (RONS) in a variety of tissues and body fluids that exceeds the physiological capacity to remove these oxygen and nitrogen species. This oxidative stress initiates damage of cell structures, including lipids and membranes, protein and DNA. The extent of oxidative stress depends on the exercise mode, intensity and duration (Bloomer, 2008). In contrast, the beneficial effects of RONS occur at low/moderate concentrations and involve physiological roles in the immune processes, cellular signalling pathways and the mitogenic response (Valko et al., 2007). RONS production is also accompanied by the upregulation of endogenous antioxidant defences. A direct proportion between exercise intensity and changes in total antioxidant capacity (TAC) has been previously reported (Parker et al., 2014). However, it seems that HIIT does not stimulate a significantly different response in TAC to responses seen following a steady-state exercise of moderate or high intensity (Wadley et al., 2016).

Since high-intensity exercise is the major factor causing impairment of muscle fibres, biochemical markers of muscle damage have also been monitored. Disruption of the sarcolemma or sarcotubular system, distortion of the contractile components of myofybrils, cytoskeletal damage and extracellular matrix changes are displays of such muscle damage (Clarkson et al., 2006), which is consequently manifested by delayed-onset pain and soreness, weakness and increases in the creatine kinase $(\mathrm{CK})$ activity, myoglobin $(\mathrm{Mb})$ and lactate dehydrogenase (LDH) in plasma (Proske and Morgan, 2001). Regarding HIIT, it has been demonstrated that short work intervals of high intensity cause greater muscle damage when compared to long submaximal intervals (Wiewelhove et al., 2015).

The acute cardiorespiratory and postexercise cardiac autonomic response to these three different single-bout HIIT sessions has been previously shown (Cipryan et al., 2016). The aim of this study was to extend these results by analysing other aspects of the exercise-induced response such as inflammation, oxidative stress and muscle damage. To the best to our knowledge, such an overall perspective on this issue, taking into account and associating numerous variables under one research design and with identical participants, has been unique thus far and may provide a genuine understanding of the physiological response to HIIT.

\section{Material and Methods}

\section{Participants}

Twelve moderately-trained males (age $22.8 \pm 1.7$ years, body height $183.9 \pm 7.8 \mathrm{~cm}$, body mass $77.0 \pm 8.4 \mathrm{~kg}$, body fat $9.9 \pm 4.0 \%, \dot{V} \mathrm{O}_{2 \max } 57.2$ $\pm 6.3 \mathrm{ml} \cdot \mathrm{kg}^{-1} \cdot \mathrm{min}^{-1}$ ) voluntarily participated in the study after receiving an explanation of the study design and signing an informed consent form. None of the participants was clinically diagnosed with any chronic or acute cardiovascular, metabolic, respiratory, immunological or musculoskeletal system disorders. Participants were non-smokers and excluded if they used any medication or dietary supplements. All the procedures received approval from the local Ethics Committee of the Ostrava University. The investigation conformed to the principles outlined in the Declaration of Helsinki.

\section{Research design}

The participants completed four experimental trials separated by a 1-2 week interval. During the first session they performed a maximal treadmill test. Three different HIIT sessions followed in randomized order. The participants were required to refrain from intense physical activity for at least $24 \mathrm{~h}$ prior to each 
session.

\section{Preliminary procedures}

In order to individually adjust the running speed for HIIT, maximum aerobic power $\left(\dot{V} \mathrm{O}_{2 \max }\right)$ and the minimal running speed required to elicit $\dot{V} \mathrm{O}_{2 \max }\left(v \dot{V} \mathrm{O}_{2 \max }\right)$ were determined by a graded exercise test (GXT). The GXT protocol began with a $3 \mathrm{~min}$ run at $8 \mathrm{~km} \cdot \mathrm{h}^{-1}$. The speed consequently increased to $12 \mathrm{~km} \cdot \mathrm{h}^{-1}$, following the exercise intensity increasing by $1 \mathrm{~km} \cdot \mathrm{h}^{-1}$ every minute up to voluntary exhaustion. A detailed description was provided previously in Cipryan et al. (2016).

\section{Exercise intervention}

The second, third and fourth experimental sessions consisted of the interval exercise interventions. All experimental sessions took place at the same time (7:00 - 9:00 a.m.). The participants always arrived at the thermally controlled laboratory after a night of fasting (i.e., no breakfast was consumed).

The HIIT interventions consisted of the following parts: (1) 8 min of a warm-up at a speed of $50 \% v \dot{V} \mathrm{O}_{2 \max } ;$ (2) interval exercise: total duration of $12 \mathrm{~min}$, work/rest ratio $=1$, work intensity $100 \% \quad v \dot{V} \mathrm{O}_{2 \max }$, rest intensity $60 \%$ $v \dot{V} \mathrm{O}_{2 \max }$, work and rest duration of $15 \mathrm{~s}, 30 \mathrm{~s}$, or 60 s; (3) a cool-down: $3 \mathrm{~min}$ at $5 \mathrm{~km} \cdot \mathrm{h}^{-1}$. All HIIT interventions were identical over the total duration, work/rest ratio, relative work and rest intensity. The heart rate and ventilatory variables (ZAN 600 Ergo; Germany) were monitored during the exercise.

\section{Monitoring of recovery}

The participants remained resting in the laboratory for $3 \mathrm{~h}$ post testing to assess the recovery process. Experienced medical staff drew blood samples from the antecubital vein with the participant in a sitting position. Venous blood was collected four times (before exercise intervention, immediately after exercise (POST), $3 \mathrm{~h}$ and $24 \mathrm{~h}$ after exercise cessation) from all the participants during all the exercise (and control) sessions. The participants were required to refrain from any exercise, alcohol drinking, keep the habitual energy intake and sleeping hours between the $3 \mathrm{~h}$ and $24 \mathrm{~h}$ blood sampling.

Fluid and food ingestion during each testing session was standardized. Each participant was accordingly provided with carbohydrate-rich, low-fat food (plain sponge biscuits $240 \mathrm{~g} ; 75.0 \mathrm{~g}$ $\mathrm{CHO}, 11.0 \mathrm{~g}$ protein and $4.9 \mathrm{~g}$ fat per $100 \mathrm{~g}$; 390 kcal per $100 \mathrm{~g}$ ) and $1.5 \mathrm{l}$ of sweet mineral water (21.4 kcal per $100 \mathrm{ml})$. It was consumed ad libidum after the exercise.

\section{Venous blood sampling and blood analysis}

The blood sample was allowed to clot for $30 \mathrm{~min}$ and subsequently centrifuged at $2000 \mathrm{G}$ for 10 minutes in order to separate the serum. The blood serum was consequently frozen at $-70^{\circ} \mathrm{C}$ until analysis. The S-Monovette ${ }^{\circledR}$ system (Sarstedt, Germany) was used for blood sample collection.

Blood samples were analysed for high sensitive IL-6 (IL-6), total antioxidant capacity (TAC), creatine kinase (CK), lactate dehydrogenase (LDH) and myoglobin (Mb). IL-6 concentrations were measured using a high sensitivity Quantikine ELISA kit (R \& D Systems, Minneapolis, MA, USA) on a DSX device (DSX, Dynex Technologies, Chantilly, VA, USA). TAC, $\mathrm{CK}$ and LDH were measured with an AU 2700 device (Beckman Coulter, Inc., Brea, CA). Myoglobin $(\mathrm{Mb})$ was measured with a Unicel Dxi 800 instrument (Beckman Coulter, Inc., Brea, CA). The analysis of IL- 6, TAC, CK, LDH and $\mathrm{Mb}$ revealed intra-assay coefficients of variation of $4.4,4.8,5.7,4.7$ and $3.8 \%$, respectively.

\section{Statistical analysis}

Collected data were checked to detect outliers and to verify sampling distribution (Shapiro-Wilk test; $p<0.05$ ). The outliers were removed and not included in the statistical analysis. The data were log-transformed using the natural logarithm if the non-normality or heteroscedasticity was revealed. All variables were presented as mean \pm standard deviation. The analysed differences were presented as standardized changes in mean (Effect size; ES). ES and $90 \%$ confidence intervals $(90 \% \mathrm{CI})$ were calculated for the between time points changes. The threshold values for ES statistics were $<0.2$ trivial, $\geq 0.2$ small, $\geq 0.6$ moderate, $\geq 1.2$ large, $\geq 2.0$ very large, $\geq 4.0$ nearly perfect (Batterham and Hopkins, 2005). The exact probabilities were expressed and the magnitude of the difference was also evaluated qualitatively as follows: $25-75 \%$ possibly, 75-95\% likely, 95-99.5\% very likely, >99.5\% most likely (Batterham and Hopkins, 2005). The smallest worthwhile change/difference was considered 0.2 of the between-individual standard deviation. If 
the $90 \%$ confidence interval overlapped small positive and negative values, the magnitude of the outcome was deemed unclear. Statistical analyses were performed using a statistical spreadsheet (Hopkins, 2006) or Microsoft Office Excel 2010 and IBM SPSS Statistics 23. The figures were made with SigmaPlot 13.0.

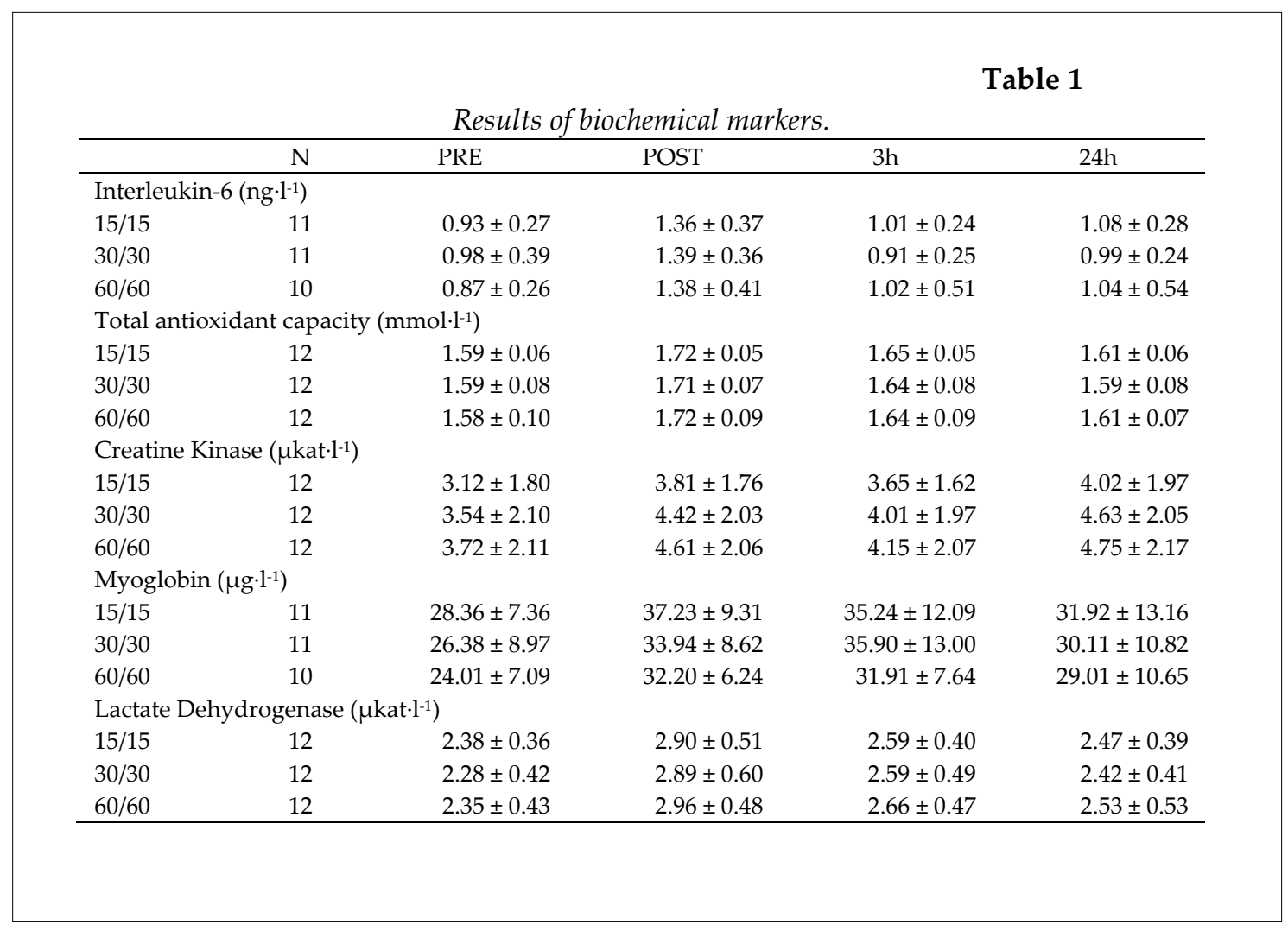

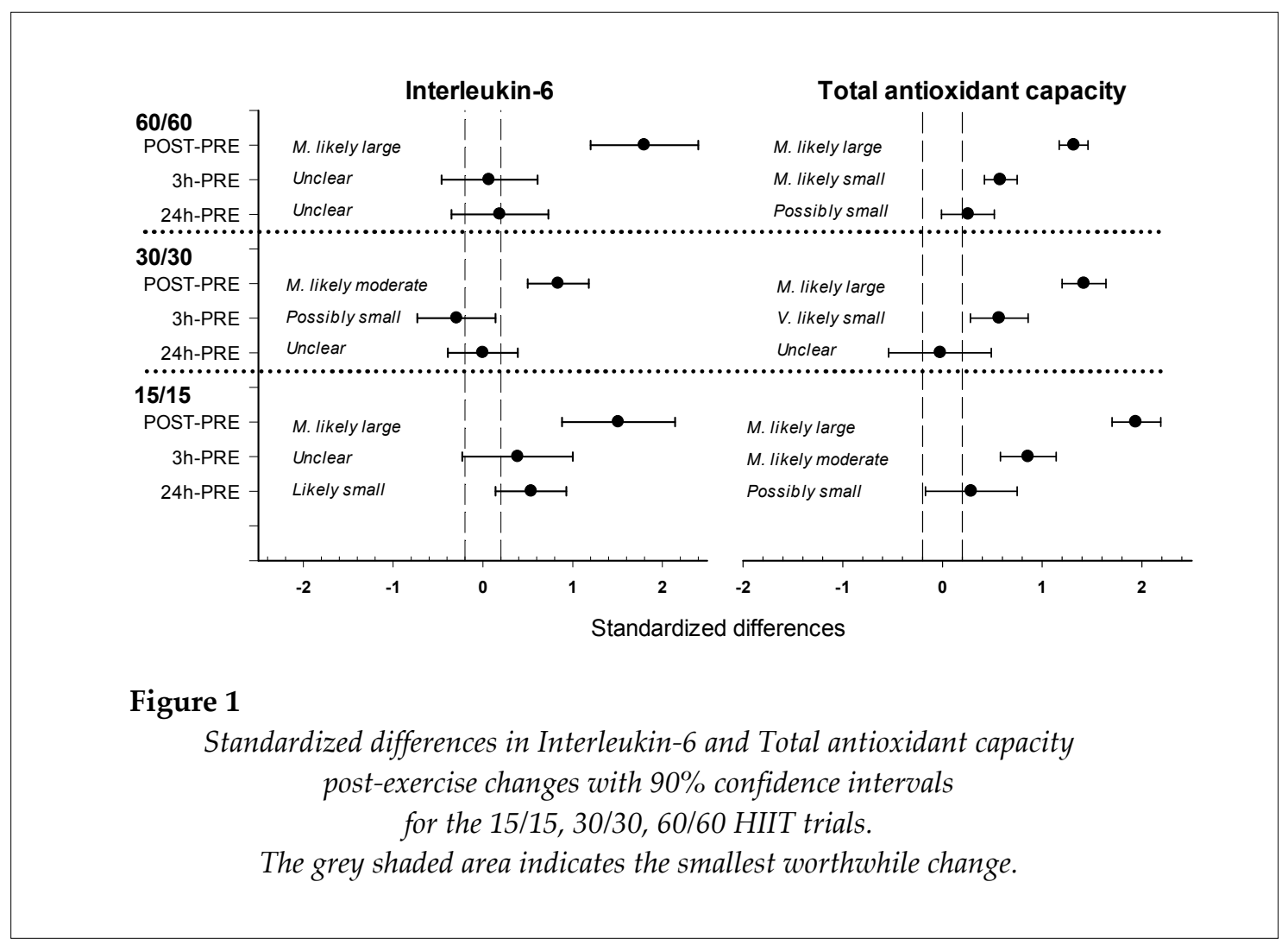



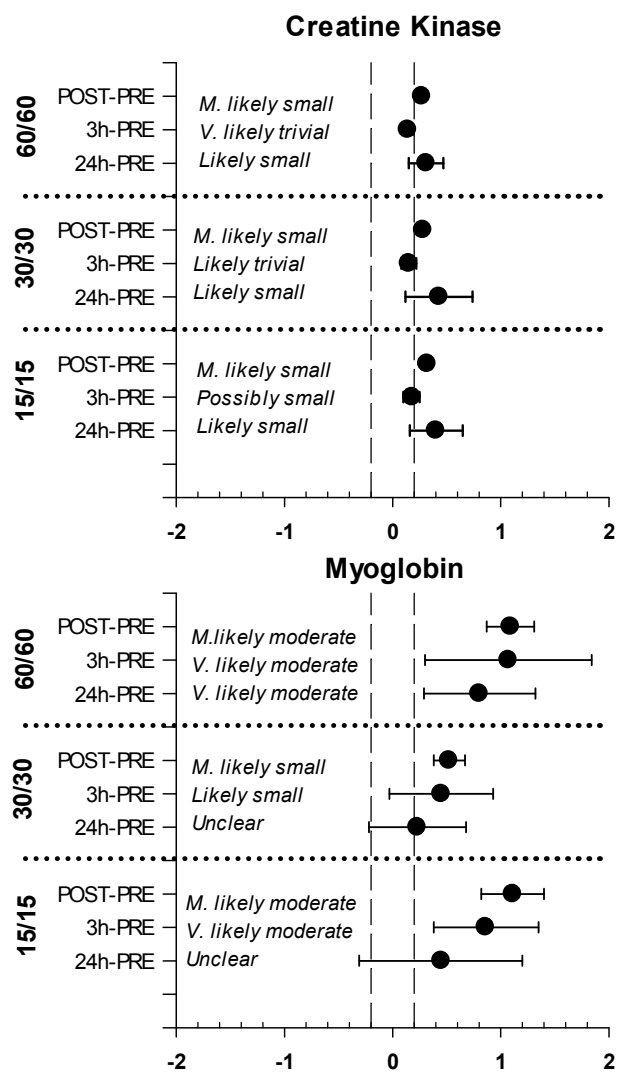

Lactate Dehydrogenase

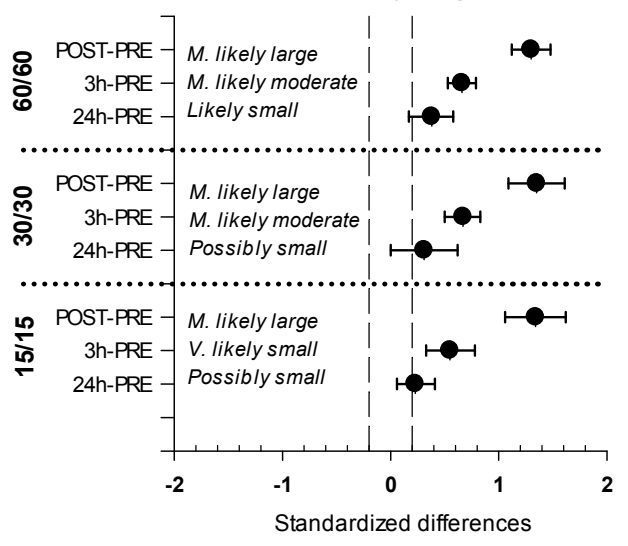

Figure 2

Standardized differences in muscle damage markers post-exercise changes with 90\% confidence intervals for the 15/15, 30/30, 60/60 HIIT trials.

The grey shaded area indicates the smallest worthwhile change. 


\section{Results}

\section{Acute cardiorespiratory response}

The acute cardiorespiratory response to the various HIIT protocols had been shown in the separate study (Cipryan et al., 2016). Briefly, there were trivial to small differences in the mean HR (15/15: $182.5 \pm 6.9 \mathrm{bpm} ; 30 / 30: 179.6 \pm 5.6 ; 60 / 60$ : $180.8 \pm 7.2 \mathrm{bpm})$, at most small differences in mean $\dot{V} \mathrm{O}_{2}\left(15 / 15: 50.2 \pm 4.2 \mathrm{ml} \cdot \mathrm{kg}^{-1} \cdot \mathrm{min}^{-1} ; 30 / 30: 49.8 \pm 3.7\right.$ $\left.\mathrm{ml} \cdot \mathrm{kg}^{-1} \cdot \mathrm{min}^{-1} ; 60 / 60: 49.5 \pm 3.6 \mathrm{ml} \cdot \mathrm{kg}^{-1} \cdot \mathrm{min}^{-1}\right)$ and unclear (30/30 vs. $60 / 60$ ) or moderate differences in RER (15/15: $0.92 \pm 0.03$; 30/30: $0.94 \pm 0.02$; 60/60: $0.94 \pm 0.03)$.

\section{Interleukin-6 (IL-6)}

All HIIT trials increased the POSTexercise IL-6 concentration. IL-6 most likely largely increased after the 15/15 (ES $\pm 90 \%$ CI: $1.51 \pm 0.63$ ) and $60 / 60$ protocol $(1.80 \pm 0.60)$, whereas the IL-6 increase after the $30 / 30$ protocol was most likely moderate $(0.84 \pm 0.34)$. Considering $3 \mathrm{~h}$ and $24 \mathrm{~h}$ measuring time points following all HIIT trials, IL-6 substantially decreased with at most moderate differences from the PRE-exercise level (Table 1, Figure 1).

\section{Total antioxidant capacity (TAC)}

TAC most likely largely increased immediately after all HIIT trials (15/15, 30/30 and 60/60: $1.94 \pm 0.24,1.42 \pm 0.22,1.32 \pm 0.15$, respectively). Subsequently, TAC markedly decreased as observed in the PRE vs. $3 \mathrm{~h}$ comparisons (15/15, 30/30 and 60/60: $0.86 \pm 0.28$, $0.57 \pm 0.29,0.58 \pm 0.16$, respectively). A possibly small increase (15/15 and 60/60: $0.29 \pm 0.46$ and $0.26 \pm 0.27)$ or unclear change (30/30: $-0.02 \pm 0.51$ ) of TAC was observed $24 \mathrm{~h}$ after the HIIT trials (Table 1, Figure 1).

\section{Creatine kinase (CK)}

The PRE vs. POST-exercise CK activity changes were most likely small considering all HIIT trials $(15 / 15,30 / 30$ and 60/60: $0.32 \pm 0.04,0.28 \pm$ $0.04,0.27 \pm 0.03$, respectively) and after the slight decrease in the PRE vs. $3 \mathrm{~h}$ comparisons (15/15, 30/30 and 60/60: $0.18 \pm 0.08,0.15 \pm 0.07,0.14 \pm 0.05$, respectively) remained likely small in the PRE vs. $24 \mathrm{~h}$ comparisons (15/15, 30/30 and 60/60: $0.40 \pm$ $0.24,0.43 \pm 0.31,0.31 \pm 0.16$, respectively) (Table 1 , Figure 2).

\section{Myoglobin (Mb)}

Changes in the $\mathrm{Mb}$ concentrations after the HIIT trials were most likely moderate in the PRE vs. POST comparisons for the $15 / 15$ and $60 / 60$ protocols $(1.11 \pm 0.29,1.09 \pm 0.22$, respectively) and most likely small for the $30 / 30$ protocol $(0.45 \pm 0.48)$. The differences remained very likely moderate (15/15: $0.86 \pm 0.48,60 / 60: 1.07 \pm 0.77)$ or likely small (30/30: $0.45 \pm 0.48$ ) in the PRE vs. $3 \mathrm{~h}$ comparisons. The $24 \mathrm{~h}$ after the HIIT trial, changes in $\mathrm{Mb}$ concentrations were very likely moderate (60/60: $0.80 \pm 0.51)$ or unclear (15/15: $0.45 \pm 0.76,30 / 30: 0.23$ \pm 0.45 ) (Table 1, Figure 2).

\section{Lactate dehydrogenase (LDH)}

LDH activity most likely largely increased after all the HIIT trials (15/15, 30/30 and 60/60: $1.34 \pm$ $0.28,1.35 \pm 0.26,1.30 \pm 0.18$, respectively). Similar changes from the pre-exercise level were observed in all HIIT protocols for $3 \mathrm{~h}(15 / 15,30 / 30$ and 60/60: $0.55 \pm 0.22,0.67 \pm 0.17,0.66 \pm 0.13$, respectively) and $24 \mathrm{~h}(15 / 15,30 / 30$ and 60/60: 0.23 $\pm 0.17,0.31 \pm 0.31,0.38 \pm 0.21$, respectively) postexercise samplings (Table 1, Figure 2).

\section{Discussion}

This study evaluated the IL-6, TAC and muscle damage responses to three different HIIT protocols of the identical external work. The major findings of the present research are that the various HIIT designs with short intervals and fixed external work caused an immediate increase in IL-6, TAC, CK, Mb and LDH. However, the $30 / 30$ protocol elicited the least IL-6 and $\mathrm{Mb}$ response while the inter-trial differences in all other analysed variables were negligible. These findings support the previously published results demonstrating the least disruption of the cardiac autonomic regulation caused by the $30 / 30$ protocol when compared to $15 / 15$ and $60 / 60$ protocols (Cipryan et al., 2016).

Interleukin-6 (IL-6)

The observed increase in IL-6 concentration after exercise, peaking at the end of exercise or early in recovery, is a consistent finding in the research literature (Pedersen and Febbraio, 2012). This fact has been previously demonstrated in response to a single bout endurance (Scherr et al., 2011) as well as highintensity interval exercise (Meckel et al., 2009; Zwetsloot et al., 2014). Since the exercise intensity influence on the IL-6 production has been already well documented (Fischer, 2006; Scott et al., 2011), it is not surprising that a superior IL-6 increase 
was observed in HIIT when compared to an exercise of moderate intensity (Wadley et al., 2016).

Increased intensity at given duration or increased duration at a given intensity during short-time exercise changes IL-6 concentration with regard to the time and amount (Wallberg et al., 2011). However, the present research investigated the influence of various HIIT protocols of both fixed variables - duration and intensity. The study results revealed inter-trial differences, which means that the exercise intensity and duration were not the only factors influencing IL-6 response to exercise. The 30/30 HIIT protocol induced the least IL-6 increase (moderate) when compared to the 15/15 (large) and $60 / 60$ (large) protocols. Moreover, it seems that the IL-6 concentration remained slightly elevated after the 15/15 protocol (Figure 1). While chronic elevation of IL-6 may induce insulin resistance, the transient IL-6 increase has a beneficial effect on insulin-stimulated glucose disposal and fatty acid oxidation (Raschke and Eckel, 2013). Therefore, the manipulation with work and rest interval duration within the HIIT prescription, even if the external work remains identical, may elicit different exercise-induced metabolic adaptation (due to a different IL-6 response to various HIIT protocols).

The IL- 6 release can be affected by the bioavailability of carbohydrates. The negative correlation between the glycogen and IL-6 level has been observed during and after the prolonged and exhausting exercise (Nieman et al., 2005). The present study did not monitor the glycogen stores and their potential depletion that might be considered a limitation of this study.

\section{Total antioxidant capacity (TAC)}

Exercise-induced oxidative stress (EIOS) may serve as a necessary stimulus for upregulation in antioxidant defences, thereby providing protection against subsequent exposure to RONS (Fisher-Wellman et al., 2009). There is evidence that endogenous antioxidant defences are adequate in maintaining redox homeostasis during short bouts of intermittent exercise in healthy individuals (Peternelj and Coombes, 2011). Findings of several studies have also confirmed that high-intensity exercise, particularly interval training, may induce beneficial redox homeostasis alterations (TAC enhancing including) and greater health benefits than low-moderate-intensity continuous exercise (Bogdanis et al., 2013; Fisher et al., 2011). However, HIIT and steady-state exercise of comparable intensity elicits similar oxidative stress and TAC response (Wadley et al., 2016).

In accordance with previous research (Parker et al., 2014), the present data provide evidence that a single bout of HIIT elicits a plasma antioxidant response. Of note, no substantial differences were observed in the magnitude of TAC between the HIIT protocols. Unlike the IL-6 response, it seems that the TAC response is HIIT protocol nondependent.

It should be mentioned that the missing direct measures of RONS limits the evaluation of the EIOS in this study. Assessment of the plasma TAC response brings only speculative information about the real EIOS. Regardless of this fact, the association between EIOS and production of antioxidant species has been previously observed (Fisher-Wellman et al., 2009). The conclusion about the redox homeostasis state and its possible disruption cannot be, therefore, definitively made.

\section{Muscle damage}

Muscle damage frequently accompanies strenuous or unaccustomed exercise. Its amount and severity depends on multiple factors, such as exercise intensity, the type of contractions, initial muscle length (Chen et al., 2007) or the force per active area (Black and McCully, 2008). The external signs of muscle damage include decreased force production, decreased range of motion, increased muscle soreness or prolonged swelling (Clarkson and Hubal, 2002). All these factors may potentially negatively influence the subsequent training session and would not be, therefore, favourable for high-frequency training. Conversely, mechanical deformation of muscle fibres may initiate specific adaptation to exercise (Spiering et al., 2008). This hypothesis has been, however, challenged as muscle rebuilding (e.g. hypertrophy) can be initiated without any discernible damage (Flann et al., 2011).

The study results revealed that all three HIIT protocols caused an elevation of muscle damage markers in circulation. However, these changes were not identical, what makes the interpretation of the muscle damage magnitude difficult. A small increase of CK activity was 
unexpected since it has been shown that HIIT is able to elicit a more extensive CK response (Wiewelhove et al., 2015). It can be explained by delayed releasing of CK into circulation. Peak CK activity had been previously observed 1-4 days after exercise and remained elevated for several days (Newham et al., 1986).

The only inter-trial differences were revealed in post-exercise $\mathrm{Mb}$ concentration and its further changes over time. Likewise IL-6, the 30/30 protocol evoked the least $\mathrm{Mb}$ increase. Therefore, the possible relationship between exercise-induced IL-6 changes and the muscle damage variables should also be discussed. It might be hypothesized that the release of IL- 6 could be a manifestation of an acute inflammatory response triggered by the accumulation of cellular debris in the areas of injury or their diffusion through the damaged sarcolemma to the interstitium and plasma (Croisier et al., 1999). It has been shown that the degree of muscle damage can be an important factor that promotes the release of IL-6 (Suzuki et al., 2006). Although the post-exercise IL- 6 and $\mathrm{Mb}$ increase was evident in all HIIT trials, particularly the $15 / 15$ and $60 / 60$ protocols, the exercise-induced changes of other muscle damage markers, i.e. CK and LDH, were not in conformity with these observations. Hence, the present findings corroborate a previous study (Mendham et al., 2011) and suggest that muscle damage is not the only factor which potentially plays an important role in regulating the acute IL6 response to exercise.

The present study compares the HIIT protocols of short-interval duration (Buchheit and
Laursen, 2013). More extensive inter-trial differences in TAC and muscle damage markers would be expected if the investigation also had involved a HIIT protocol of long-interval duration. The acute metabolic and cardiorespiratory responses of short-interval HIIT are similar to constant load exercise and substantially lower than in long-interval HIIT if matched by the mean load and total duration (Tschakert et al., 2015). Therefore, sport practitioners should be aware that even this slight manipulation with work interval duration (within the short HIIT format) may cause a different physiological response.

\section{Conclusions}

The present investigation showed that a various design of HIIT protocols might be considered important for physiological adaptation, even if the total external work is identical. Since the beneficial effect of IL- 6 on the muscle metabolism had been shown (Raschke and Eckel, 2013), our results indicated that the $15 / 15$ and $60 / 60$ protocols might be preferred to the $30 / 30$ protocols in order to maximize the training stimulus. These findings are in conformity with the sister paper, which showed a similar acute cardiorespiratory response to all three HIIT protocols as well as the least disturbance of cardiac autonomic regulation following the 30/30 HIIT protocol (Cipryan et al., 2016).

\section{Acknowledgements}

This study was supported by Ostrava University under Grant SGS12/PdF2013. The author kindly thanks Vaclav Kana for assisting during data collection, Dr. Zdenek Svagera for the contribution with a biochemical analysis and Prof. Peter Hofmann for valuable comments on an earlier draft.

\section{References}

Batterham AM, Hopkins WG. Making meaningful inferences about magnitudes. Sportscience, 2005; 9: 6-13

Bernecker C, Scherr J, Schinner S, Braun S, Scherbaum WA, Halle M. Evidence for an exercise induced increase of TNF- $\alpha$ and IL-6 in marathon runners. Scan J Med Sci Sports, 2013; 23: 207-214

Black CD, McCully KK. Muscle injury after repeated bouts of voluntary and electrically stimulated exercise. Med Sci Sports Exerc, 2008; 40: 1605-1615

Bloomer RJ. Effect of exercise on oxidative stress biomarkers. Adv Clin Chem, 2008; 46: 1-50

Bogdanis GC, Stavrinou P, Fatouros IG, Philippou A, Chatzinikolaou A, Draganidis D, Ermidis G, Maridaki 
M. Short-term high-intensity interval exercise training attenuates oxidative stress responses and improves antioxidant status in healthy humans. Food Chem Toxikol, 2013; 61: 171-177

Buchheit M, Laursen PB. High-intensity interval training, solutions to the programming puzzle - Part II: Anaerobic energy, neuromuscular load and practical applications. Sports Med, 2013; 43: 927-954

Chen TC, Nosaka K, Sacco P. Intensity of eccentric exercise, shift of optimum angle, and the magnitude of repeated-bout effect. J Appl Physiol, 2007; 102: 992-999

Cipryan L, Laursen PB, Plews DJ. Cardiac autonomic response following high-intensity running work-torest interval manipulation. Eur J Sport Sci, 2016; 16: 808-817

Clarkson P, Hubal M. Exercise-induced muscle damage in humans. Am J Phys Med Rehab, 2002; 81: S52-S69

Clarkson PM, Kearns AK, Rouzier P, Rubin R, Thompson PD. Serum creatine kinase levels and renal function measures in exertional muscle damage. Med Sci Sports Exerc, 2006; 38: 623-627

Croisier JL, Camus G, Venneman I, Deby-Dupont G, Juchmes-Ferir A, Lamy M, Crielaard JM, Deby C, Duchateau J. Effects of training on exercise-induced muscle damage and interleukin 6 production. Muscle \& Nerve, 1999; 22: 208-212

Febbraio MA, Pedersen BK. Muscle-derived interleukin-6: mechanisms for activation and possible biological roles. The FASEB J, 2002; 16: 1335-1347

Fischer CP. Interleukin-6 in acute exercise and training: what is the biological relevance? Exerc Immunol Rev, 2006; $12:$ 6-33

Fisher G, Schwartz DD, Quindry J, Barberio MD, Foster EB, Jones KW, Pascoe DD. Lymphocyte enzymatic antioxidant responses to oxidative stress following high-intensity interval exercise. J Appl Physiol, 2011; 110: 730-737

Fisher-Wellman K, Bell HK, Bloomer RJ. Oxidative stress and antioxidant defence mechanisms linked to exercise during cardiopulmonary and metabolic disorders. Oxid Med Cell Longev, 2009; 2: 43-51

Flann KL, LaStayo PC, McClain DA, Hazel M, Lindstedt SL. Muscle damage and muscle remodelling: no pain, no gain? J Exp Biol, 2011; 214: 674-679

Heydari M, Boutcher YN, Boutcher SH. High-intensity intermittent exercise and cardiovascular and autonomic function. Clin Auton Res, 2013; 23: 57-65

Tschakert G, Kroepfl J, Mueller A, Moser O, Groeschl W, Hofmann P. How to regulate the acute physiological response to "aerobic" high-intensity interval exercise. J Sports Sci Med, 2015; 14: 29-36

Hopkins WG. Spreadsheets for analysis of controlled trials, with adjustments for a subject characteristic. Sportscience, 2006; 10: 46-50

Meckel Y, Eliakim A, Seraev M, Zaldivar F, Cooper DM, Sagiv M, Nemet D. The effect of a brief sprint interval exercise on growth factors and inflammatory mediators. J Strength Cond Res, 2009; 23: 225-230

Mendham AE, Donges CE, Liberts EA, Duffield R. Effects of mode and intensity on the acute exerciseinduced IL-6 and CRP responses in a sedentary, overweight population. Eur J Appl Physiol, 2011; 111: 1035-1045

Milanović Z, Sporiš G, Weston M. Effectiveness of high-intensity interval training (HIT) and continuous endurance training for $\mathrm{VO}_{2 \max }$ improvements: a systematic review and meta-analysis of controlled trials. Sports Med, 2015; 45: 1469-1481

Newham DJ, Jones DA, Edwards RHT. Plasma creatine kinase changes after eccentric and concentric contractions. Muscle \& Nerve, 1986; 9: 59-63

Nieman DC, Davis JM, Henson DA, Gross SJ, Dumke CL, Utter AC, Vinci DM, Carson JA, Brown A, McAnulty SR, McAnulty LS, Triplett NT. Muscle cytokine mRNA changes after $2.5 \mathrm{~h}$ of cycling: influence of carbohydrate. Med Sci Sports Exerc, 2005; 37: 1283-1290

Pal M, Febbraio MA, Whitham M. From cytokine to myokine: the emerging role of interleukin-6 in metabolic regulation. Immunol Cell Biol, 2014; 92: 331-339

Parker L, McGuckin TA, Leicht AS. Influence of exercise intensity on systemic oxidative stress and 
antioxidant capacity. Clin Physiol Funct I, 2014; 34: 377-382

Pedersen BK, Febbraio MA. Muscle, exercise and obesity: skeletal muscle as secretory organ. Nat Rev Endocrinol, 2012; 8: 457-465

Peternelj TT, Coombes JS. Antioxidant supplementation during exercise training: beneficial or detrimental? Sports Med, 2001; 41: 1043-1069

Proske U, Morgan DL. Muscle damage from eccentric exercise: mechanism, mechanical signs, adaptation and clinical applications. J Physiol, 2001; 537: 333-345

Raschke S, Eckel J. Adipo-Myokines: Two Sides of the Same Coin-Mediators of Inflammation and Mediators of Exercise. Mediat Inflamm, 2013; doi:10.1155/2013/320724

Scott JPR, Sale C, Greeves JP, Casey A, Dutton J, Fraser WD. Effect of Exercise Intensity on the Cytokine Response to an Acute Bout of Running. Med Sci Sports Exerc, 2011; 43: 2297-2306

Scherr J, Braun S, Schuster T, Hartmann C, Moehlenkamp S, Wolfarth B, Pressler A, Halle M. 72-h kinetics of highsensitive troponin T and inflammatory markers after marathon. Med Sci Sports Exerc, 2011; 43: 1819-1827

Spiering BA, Kraemer WJ, Anderson JM, Armstrong LE, Nindl BC, Volek JS, Maresh CM. Resistance exercise biology manipulation of resistance exercise programme variables determines the responses of cellular and molecular signalling pathways. Sports Med, 2008; 38: 527-540

Suzuki K, Peake J, Nosaka K, Okutsu M, Abbiss CR, Suriano R, Bishop D, Quod MJ, Lee H, Martin DT, Laursen PB. Changes in markers of muscle damage, inflammation and HSP70 after an Ironman Triathlon race. Eur J Appl Physiol, 2006; 98: 525-534

Tschakert G, Kroepfl J, Mueller A, Moser O, Groeschl W, Hofmann P. How to regulate the acute physiological response to "aerobic" high-intensity interval exercise. J Sports Sci Med, 2015; 14: 29-36

Valko M, Leibfritz D, Moncol J, Cronin MTD, Mazur M, Telser J. Free radicals and antioxidants in normal physiological functions and human disease. Int J Biochem Cell B, 2007; 39: 44-84

Yudkin JS. Inflammation, obesity, and the metabolic syndrome. Horm Metab Res, 2007; 39: 707-709

Wadley AJ, Chen Y-W, Lip GYH, Fisher JP, Aldred S. Low-volume-high intensity interval exercise elicits antioxidant and anti-inflammatory effects in humans. J Sports Sci, 2016; 34: 1-9

Wadley AJ, Van Zanten JJCS, Aldred JCS. The interactions of oxidative stress and inflammation with vascular dysfunction in ageing: The vascular health triad. Age, 2013; 35: 705-718

Wallberg L, Mattson CM, Enqvist JK, Ekblom B. Plasma IL-6 concentration during ultra-endurance exercise. Eur J Appl Physiol, 2011; 111: 1081-1088

Wiewelhove T, Fernandez-Fernandez J, Raeder C, Kappenstein J, Meyer T, Kellmann M, Pfeiffer M, Ferrauti A. Acute responses and muscle damage in different high-intensity interval running protocols. J Sports Med Phy Fitness, 2015; PMID: 25665748

Zweetsloot KA, John C, Lawrence M, Battista RA, Shanely RA. High-intensity interval training induces a modest systemic inflammatory response in active, young men. Journal of Inflammation Research, 2014; 7: $9-17$

\section{Corresponding author:}

\section{Lukas Cipryan, Ph.D.}

University of Ostrava

Varenska 40a

70200 Ostrava

Czech Republic

Email: lukas.cipryan@osu.cz

Phone: +420605807925

Twitter:@LukasCipryan 